Mikkel B. Tin

\title{
Making and the sense it makes
}

Making is ubiquitous, and it is as ancient as culture. In fact, making is the practical dimension of culture. It transforms matter, and it articulates meaning. Making has a cognitive dimension; it makes sense. But this sense is not ordinary discursive knowledge - making yields another kind of knowledge, often referred to as 'tacit' because it seems to go without saying. Now, if it is tacit how can we speak about it, and what is its role in making?

During the last decades, we have witnessed, perhaps also contributed to, the appearance of making on the academic arena. It has been met with a great deal of scepticism on the side of the institutions - yet studies such as architecture, design and arts, generally acknowledged as 'making disciplines', have proved to be researchable, and the interest they arouse keeps growing. The present series of articles based on contributions to the Making conference at Notodden, Norway, in September 2012, is one among several indications that making is increasingly recognised as 'an emergent field of study'.

But what exactly does it mean for a human activity to be recognised as a 'field of study'? This wording may refer to at least three quite different epistemological situations that I will discuss briefly: 1) Making seen as a study, or inquiry, in its own right, independently of scientific research, as a source of non-scientific cognition; 2) making as the object of study within academic research, as the object of scientific cognition, and 3) making as an integrated part of academic research, as a source of scientific cognition redefined.

\section{Making as a source of non-scientific cognition}

Making, obviously, is practical, yet we may agree that there is a cognitive potential in its approach as well as its results. Making certainly need not be artistic creation, though it sometimes is; but in order for it to deserve its name we expect it to be more than mechanical production. It consists in an intentional forming process whose outcome is articulated meaning.

This forming process involves my body and is performed as a bodily practice. But even as a bodily practice it requires training, and certain kinds of professional making require a long and comprehensive training. Still, the practical skill that results from such training is bodily rather than conceptual. The training and transmission of making skills take the form of practical experimentation rather than theoretical explanation. The aim is a concrete solution to a given problem, not an abstract investigation into a general problem. We have experienced how fully we must focus on our body and immediate surroundings when learning even the relatively simple art of, say, a lino cut, and how complicated, if not impossible, this art would be if taught to us in lessons of kinetics and physics. If we were to understand our art theoretically, without ever trying it out practically, we would never acquire the Fingerspitzengefühl $^{l}$ that is the prerogative of the accomplished maker. This explains the longevity of the master-apprentice teaching method based on copying, 'learning by doing' and embodied know-how.

Since ancient Greece we distinguish between practical and theoretical knowledge, techne and episteme. This distinction accounts for the division between practical and theoretical studies, and the two kinds of institutions in which these studies have been carried out according to very different didactic principles. And we all know how far the making disciplines have reached within their own educational system and professional environment: in workshops and studios, academies and conservatories, schools of architecture, technical universities. That is where, until very recently, all outstanding achievements within applied 
and fine art, music, dance, architecture, town planning, engineering, design have been conceived and produced, far from the concerns of science and theoretical research.

Even though many such achievements are comparable to, though different from, scientific research, there are good reasons for practical research to keep its distance from science: the constraints imposed by scientific rationality, such as systematicity, conceptuality, reflectivity, reproducibility, may hinder the truly creative endeavour, and the absence of tools and materials in theoretical studies obviously impede the acquisition of the making skills.

\section{Making as the object of scientific cognition}

Making may open various fields of study to scientific research and, as the important cultural phenomenon it is, definitely deserves to be studied. But scientific research is based on the claim of objectivity, and objectivity presupposes a clear distinction between subject and object. The ideal is a distanced, impartial viewpoint, both in the description of empirical material, in carrying out controlled experiments, and in the interpretation of data generated. As the object of scientific research, making therefore can no longer be studied from the insider's perspective only, it must be approached from outside, like the polar bear in zoology, or kinship in social anthropology. It will be analysed and conceptualised, following the same principles as in anatomy, art history, sociology, psychology or whatever. The method is inductive: observation of particular cases, and then establishment of general features through description and interpretation according to appropriate theoretical grids. The result is propositional knowledge.

From being performed - unnoticed by, and independent of, scientific research making now tends to appear as an object of scientific research. But what becomes of making in scientific research if making is essentially non-conceptual and scientific research is essentially conceptual? Will making be allowed to appear as a searching approach in its own right, yielding insights that a purely intellectual approach does not? Or is there a danger that making, as the object of scientific research, will be reduced to its mechanical aspects, material results, social preconditions, or other contingencies?

Such dangers have beset other human, medical and cultural phenomena in scientific research. When women or mental patients or ethnic groups or, for that matter, the third world, were introduced as fields of study, they ended up in an asymmetrical relationship as merely the object on which research is carried out by a subject who initiated and undertook the research. Whatever format making may have had originally, it will be re-formatted as an object according to the requirements and rationality of scientific research. In this manner it may be deprived of the special intentionality that enlivens it inherently.

The recognition of the making practices as 'emergent fields of study' thus involves at least two major dangers: to disregard the non-conceptual aspect of making and to overlook its intentional character.

\section{Making as a source of scientific cognition redefined}

In order to be scientific, research must attain a validity that reaches beyond the concrete and private features of the endeavour. If we agree on this point we must also recognise that the concreteness of making, which is its strength in practical life, becomes its weakness in scientific research. The artefacts that result from making are particular, not general; and the meaning they articulate is specific rather than typical. In that sense, making exceeds the scientific paradigm.

On the other hand, we have the right to question the apparent evidence of the scientific paradigm and the requirements it imposes on research. The self-contained and ultimately selfsufficient character of much scientific research may turn out to be its fatal weakness. From 
this perspective, the criteria of validity that apply to the making inquiry, namely its practical relevance, may be beneficial for theoretical enquiry too.

We know that this paradigm, in spite of the pretentions of scientific institutions, has undergone substantial change throughout history, and that there is no such thing as eternal truth or criteria of truth, not even in science. The positivist rationality that seemed absolute in most disciplines during the $19^{\text {th }}$ century is now regarded as obsolete. Not only did it turn out to be inappropriate for the emergent humanities and social sciences, but the entire idea of objectivity began to reel once the sovereign subject had been challenged by its own embodiment. Once essence was re-rooted in existence and language, consequent claims held no more than conditional significance.

The making disciplines can complement the scientific paradigm in useful ways. They open the dimensions of embodiment and materiality as an inherent aspect of understanding. Nowhere will we ever find the position of the impartial observer; but the situation to which our own embodiment and the materiality of the world confine us helps us understand making - and existence generally - as constant efforts to reach from the given to the possible. Making is a form of research, in as far as it pursues a goal that is sufficiently clear to be perceived, but sufficiently unclear still to deserve to be pursued in and through making.

The making disciplines also visualise the processual aspect of understanding - which takes the shape of an on-going dialogue between subject and object. In fact, all making is not only a dialogue between the forming will and the material conditions; it is interaction and gradual interpenetration. Meaning is not prior to, nor independent of, our meaningful articulation. It is only in and through articulation that meaning appears. Meaning is not given, it appears through making.

Making is a source of cognition, but in order for it to be recognised as a source of scientific cognition, both the practical techne of the making disciplines, and the theoretical episteme of the scientific paradigm ought to approach the more ethical and situational understanding of phronesis.

\section{Making an epistemological difference}

The history of science takes account of a series of struggles fought by emerging research fields in order to gain recognition: the humanities, the social sciences, psychology. At best, scientific research carries out critical self-reflection along with the critical scrutiny of its objects. The scepticism with which it meets new-comers to the field may be salutary in that it forces the latter to reflect on their scientific pretensions. In order to strengthen their disciplinary identity, even the making disciplines would benefit from a far more rigorous and distinctive definition than we see today.

There definitely are multiple ways to make: from blacksmithing to BioArt, from crafts in elementary schools to information technology, from classical dance to city planning. And not all of their activities display such qualities as would make them apt sources of scientific cognition. On the other hand, they do in certain cases represent the kind of phronesis that is needed in innovative scientific research - not only for the mastery of techniques and materials, but also to be able to comprehend man as embodied intentionality, and life as his making encounter with materiality.

As already stated, scientific research is, in a sense, nothing but conceptual generalisation: So even the making disciplines must develop an operational set of concepts in order to clarify their focus and facilitate their mutual understanding. We need to define concepts such as embodiment, bodily skills, materiality, knowledge, tacit knowledge, practice and creation. We must discuss the making procedures and the ways to assess the products of making. 
But we also need definitions of making itself, the common denominator of the making disciplines, in as far as they can be integrated into scientific research, not just as study objects but as research methods sui generis. In view of such a definition, and to fuel the ongoing discussion, I will risk a few answers to the question: 'What distinguishes making as research?'
1. It is an explorative and creative process.
2. It is intentional but engages the skilled body.
3. It follows an ordered sequence of experiments and reflections.
4. It forms and transforms materiality.
5. It generates a specific knowledge.
6. It articulates a meaning that can only partly be conceptualised.

In addition to these aspects that can be seen to distinguish making as research method, making must of course comply with the same general requirements as all other scientific research such as:

\section{Clearly formulated premises and research questions.}

2. Adequate methods and theoretical tools to answer the research questions, including reproducibility and documentation.

\section{Adequate tools for self-criticism and impartial assessment.}

\section{Adequate format for presentation and assessment.}

5. Communicable and transferable results, that are also compatible with other disciplines.

\section{Institutional integration.}

Existing research fields like medicine or chemistry have the privilege at their disposal of a well-established disciplinary discourse, including a recorded history, a set of theoretical and critical tools. They have also at their disposal, hospitals and laboratories where, in researchers' communities, they develop and communicate their research. They have their scientific journals and international conferences. Increasingly, the results of provocative thinking and analytical research approaches within the making community are being discussed at international conferences and published in newly recognised high quality journals. Progressively, more and more discursive and interactive space is becoming available for the making professions to articulate and challenge the knowledge of making. Making itself is an articulation of meaning and part of its potential resides in its creation of new means and forums for communication. The making disciplines, therefore, also provide a far-reaching reflection on the essence of meaning and communication that can be valuable to all kinds of research. 


\section{Mikkel B. Tin}

Professor of Traditional Arts

Department of Folk Art and Folk Music, Telemark University College.

E-mail address: mikkel.b.tin@hit.no

\section{How to quote this article:}

Tin, M.B. (2013) Making and the sense it makes. Conceptual manifesto simultaneously published in FORMakademisk, Vol 6:2, Studies in Material Thinking, Vol 9, and Techne A Series, Vol 20:2, and the URL for either publications.

\footnotetext{
${ }^{1}$ Fingerspitzengefühl is a German word that refers to the embodied judgement concerning material handling and manual execution.
} 